\title{
基于雨课堂的任务导向式化学专业英语教学模式探索与实践
}

蒋达洪 ${ }^{*}$, 姚晓青, 黄艳仙, 肖朵朵

广东石油化工学院化学学院, 广东茂名 525000

摘要: 针对传统化学专业英语教学中存在的问题, 提出将任务导向与雨课堂相结合的教学模式运用到专业英语课程 的教学中。分析了任务导向与雨课堂相融合的特点, 阐述了在专业英语教学中实施以雨课堂为基础的任务导向式教 学环节与方法, 利用问卷调查和定性对比方式考查了教学效果。结果发现, 该教学模式能够显著地提高课堂气氛、 师生互动度和学生参与度; 融合雨课堂能显著提高任务教学的效果; 雨课堂能一定程度地改善日常教学管理; 该教 学模式对学生兴趣和学业成绩的提高具有促进作用。

关键词: 雨课堂; 任务导向; 教学模式; 化学专业英语

中图分类号: G642; O6

\section{Study and Practice on Teaching Mode of Specialized English for Chemistry Merging Task-Based Method with Rain Classroom}

\author{
Dahong Jiang ", Xiaoqing Yao, Yanxian Huang, Duoduo Xiao \\ College of Chemistry, Guangdong University of Petrochemical Technology, Maoming 525000, Guangdong Province, P. R. China.
}

\begin{abstract}
In response to the problems existing in the traditional English for chemistry teaching, a new mode is proposed merging task-based method with rain classroom. The characteristics of the integration of task orientation with rain classroom are analyzed and the teaching procedure, methods and effects in the course are described. Questionnaire survey and qualitative comparison are used to examine the effect of this teaching mode. The results show that the teaching mode can significantly improve the classroom atmosphere, teacher-student interaction and student participation; the integration of rain classroom can significantly improve the effect of task-based teaching; rain classroom can improve the daily teaching management to a certain extent; and the teaching mode can improve students' interest as well as academic performance.
\end{abstract}

Key Words: Rain classroom; Task-based; Teaching mode; Specialized English for chemistry

化学作为一门中心学科, 在当今世界频繁的科技交流和商务往来中地位日益突出 ${ }^{[1]}$ 。然而长久 以来, 我国都大量短缺具有较高英语交流能力的复合型化学化工类人才, 这已经成为化学专业英语 教学所面临的一个 “痛点” 。在大多数教师眼中, 化学专业英语教学是一块难啃的 “硬骨头”。它 作为化学与英语的跨学科交叉, 不仅要教给学生大量专业词汇和特定的表达, 更重要的是能运用英 语作为工具去获取所需的专业知识和进行专业交流 ${ }^{[2]}$ 。因此, 英文应用能力的培养亦是教学的重要 组成部分。然而, 担任化学专业英语教学的教师一般都是化学专业出身, 对外语教学法并无深入的 研究 ${ }^{[3]}$ 。课堂上大多数采用 “一言堂” 式的翻译教学法, 即首先讲解新单词的汉语意思, 然后对课

收稿: 2019-06-20; 录用: 2019-07-13; 网络发表: 2019-08-20

“通讯作者, Email: jiangdh@gdupt.edu.cn

基金资助: 广东省本科高校高等教育教学改革项目(2017396) 
文再逐字逐句进行翻译。这种教学模式虽然有利于学生在较短的时间内掌握专业英语词汇, 提高翻 译水平, 但是师生互动少、课堂比较沉闷枯燥、学生难以跟上教师节奏而丧失学习兴趣 ${ }^{[4,5]}$ 。要克服 这些弊端, 必须寻找教学模式方面的创新。

随着移动互联网技术的发展, 新的教学手段不断涌现。其中, 清华大学推出的雨课堂教学手段 极大地促进了教与学的交流互动 ${ }^{[6]}$, 在许多学科教学中得到广泛应用。充分利用现代教育技术、借 鉴语言教学理论, 探索提高化学专业英语的教学质量显得非常迫切。任务导向型教学模式原本是 20 世纪 80 年代在研究第二语言习得理论中提出的一种语言教学法, 它强调以学生为主体, 围绕任务的 解决营造自主学习氛围, 通过互动、愉悦的课堂平台提高外语教学质量 ${ }^{[7,8]}$ 。本文借鉴这一理论, 运 用雨课堂教学手段构建了以完成任务为目标的新型化学专业英语教学模式, 继而将这一模式应用于 教学实践, 提高了学生专业英语水平和运用英语获取知识的能力, 使传统教学过程中的被动式学习 转变为主动式学习。

\section{1 任务导向与雨课堂在专业英语教学中的特点}

\section{1 任务导向在专业英语教学中的特点}

化学专业英语对于刚刚接触的学生来说比较陌生, 一方面学生对能够把学了十年的英语应用在 专业知识的获取上感到兴奋, 另一方面又感到畏惧而容易失去信心。在教学模式上应该紧紧抓住学 生的这种兴奋点, 摒弃传统的以 “灌输知识” 为主的教学模式, 根据学生接受知识能力的差别和实 际交流的需要, 转变为以任务为目标、以 “子任务” 为模块的教学形式。通过精心设计和组织教学, 使之符合学生的认知特点, 着重培养学生自主学习能力和灵活解决问题的能力。

任务导向式教学模式承认学生的个体差异, 教师根据学生能力的不同, 灵活分配相应的任务。 对于英语基础强的学生, 应当着重培养其独立获取化学专业知识的能力, 拓展其思维; 英语基础稍 弱的学生, 教师在分配任务时, 应重基础, 提高其专业英语水平。

\section{2 雨课堂在专业英语教学中的特点}

首先, 雨课堂不局限在课内, 还包括课外部分。教师可在课前制作出关于专业英语教学中各个 知识点的预习材料, 包括 PPT、视频、语音、习题等。学生课下预习后可在课堂上或雨课堂讨论区 与教师和同学进行交流, 教师针对学生的困惑和不解之处进行讲解。教师还可以根据回放实时记录, 跟踪了解学生的学习情况, 这是一种教师与学生之间互动交流的教学模式。这一模式合理配置了学 生的课余时间, 极大地提高了知识传授与接受的效率。

其次, 雨课堂提高了学生学习的自主性和灵活性, 通过高效预习, 教师和学生能在课堂中畅所 欲言地交流, 把握重点; 同时雨课堂又是一种多向互动的教学模式, 学生可通过网上或课堂上同教 师、其他同学互动, 教师还可以照顾学习有困难的学生, 提高学生的学习效率。

\section{3 任务导向与雨课堂在专业英语教学中相融合的特点}

雨课堂和任务导向教学手段在我校其他课程的教学中已有开展。实践表明, 仅仅单一使用任务 导向或雨课堂的效果并不很明显, 没有任务导向的雨课堂仅仅是将课下学习从 “纸质版” 变为 “电 子版” ，而没有雨课堂的任务导向则容易因缺少互动与指导而半途而废。教师应该设计出更好的方 法使两者相互融合, 实现优势的互补和叠加。研究发现, 任务导向是雨课堂的关键, 设计出好的 “子 任务” 优势明显, 这能够让学生按照自己的知识水平自主地安排学习。实施任务导向, 既能帮助教 师有效运用雨课堂教学手段, 转变教学模式, 提高教学质量培养创新型复合人才, 又能帮助学生合 理安排学习时间自主学习, 提高学习效率。

\section{2 基于雨课堂的任务导向在专业英语教学中的组织与实施}

传统教学中, 课堂主体通常是教师, 如果教师不能掌握学生的学习进程, 将严重影响教学效果。 在新的教学模式中, 雨课堂与任务导向相结合, 主体反转为学生, 教师的主要任务是充当 “组织者”, 
即指导学生如何去学和激发学习兴趣, 而不是灌输知识。学生可以针对教师发布的任务, 自主进行 学习, 这样就可以充分调动学生学习的主动性和积极性, 提高学习热情。雨课堂和任务导向都有课 前、课中、课后、评价 4 个阶段, 它们有着相似的教学理念, 将二者有机结合起来运用到化学专业 英语教学中, 能够转变传统的教学模式, 使沉闷的课堂变得生动活泼。下面以 “The Nomenclature of Inorganic Compounds” 的教学为例, 阐述其组织与实施。

\section{1 任务推送：课前预习环节}

首先将班级学生按照英语基础水平的不同分为 $\mathrm{A} 、 \mathrm{~B} 、 \mathrm{C}$ 三组, 每组十人左右, 指定小组长。课 前一到两天, 教师将准备好的预习材料通过雨课堂平台推送给学生, 并给每组学生布置若干个子任 务, 设置在规定时间内完成预习。学生自主预习时遇到不懂的问题可以在雨课堂讨论区报告教师, 教师及时进行一对一指导。教师也可以实时查看学生的预习情况, 根据 “子任务” 的初步解决情况 对授课方式和内容进行调整, 明确教学重点与难点。学生也能够带着任务有针对性地听课, 对相关 知识点进行巩固。本课程推送材料与子任务示例见表 1 。

表 1 课前预习环节的任务推送

\begin{tabular}{|c|c|c|}
\hline 组别 & 共用预习材料 & 分组子任务 \\
\hline A & \multirow{4}{*}{$\begin{array}{l}\text { 隐去课堂习题的课件 PPT } \\
\text { 教师录音材料: } \\
\text { 1) 英文表示数字的希腊词头解释与读音; } \\
\text { 2) IUPAC 命名原则 }\end{array}$} & 用英文列表总结二元化合物、酸、盐命名通则 \\
\hline B & & 团队协作翻译离子化合物的命名部分 \\
\hline \multirow[t]{2}{*}{$\mathrm{C}$} & & 1) 朗读课后专业词汇; \\
\hline & & $\begin{array}{l}\text { 2) 举例说明词头 per-, hypo-, 词尾-ite, -ate, -ide, 以及-ic, } \\
\text {-ous 在命名中的差别 }\end{array}$ \\
\hline
\end{tabular}

教师设计任务时要注意把握难度, 根据每组学生能力, 布置推送相对应难度的子任务。本环节 要求学生先结合课本完成预习材料, 然后初步解决推送的子任务。对于团队协作项目, 由小组长负 责组织分工共同完成并标明每位学生的贡献。

\section{2 任务解决: 课堂互动环节}

课堂上, 教师首先利用微信, 通过雨课堂平台自动生成二维码, 学生扫码进入课堂。进入后, 本节课的 PPT 会实时显示在学生手机上, 学生对不懂之处可利用 “不懂” 按钮进行标记, 与教师互 动。教师根据 “不懂” 人数情况及时调整授课内容和重点。教师还可以通过弹幕实现实时互动, 学 生可以将自己的观点或问题通过弹幕与教师和其他同学分享, 促进多边互动, 这特别给比较腼腆内 向的学生以发言机会。

在解决任务时, 通过小组讨论、总结发言等传统方式, 由每组选择 2-3 名代表就子任务进行讲 解, 其余学生聆听并进行补充。此外, 教师还可以通过雨课堂设置习题与投票来完成任务讨论, 题 型包括单选、多选、填空、主观题等, 答题结果会实时反馈给教师和全班同学。这样, 通过师生的 讨论、学习使各个子任务得到圆满解决。在解决任务后, 教师顺水推舟地对教材中的重点知识进行 讲解。如利用 $\mathrm{A}$ 组的任务解决方案, 归纳出非金属二元化合物、离子化合物、无机酸、盐、水合物 的英文命名。作为专业英语, 教师应 “内容优先、兼顾语言” , 对词汇和语法的讲解目的是理解内 容。

\section{3 任务完成: 课后总结环节}

课堂结束后, 教师手机会收到课后小结, 具体到哪位学生没有圆满解决任务。教师通过分析课 后小结数据, 结合学生课堂表现进行分析, 对教学进行总结与反思。一方面, 教师可通过总结改进 教学方法, 完善教学模式; 另一方面, 根据学生完成任务的情况, 判断学生哪些知识点尚未完全掌 握, 再补充推送课后习题, 帮助学生消化知识点, 及时复习巩固。在课后的复习中, 学生有任何问 
题都可以点击 “报告教师”, 使教与学永远在线。

例如, 通过数据分析, 发现部分学生混淆了各类化合物命名方法。教师精选推送含不同种类化 合物的 “(1) name the following compounds; (2) write the formula” 习题作为雨课堂课后作业, 并设置 完成后显示解答过程, 使学生及时得到反馈。

为了激发学生进一步的学习兴趣, 教师精选网络中开放的英文文献作为选学内容, 在雨课堂公 告栏中以分享链接方式引导学生阅读, 让学生学以致用。

\section{4 任务评价：成绩考核环节}

传统的课程考核方式主要依靠期末考试 “一锤定音”，这不仅使考核成绩因学生临场发挥而增 加偶然性，还变相鼓励学生平时不用功而考前临时抱佛脚，不利于专业英语水平的提高。

雨课堂支持的智慧课堂利用大数据技术, 对每一个学生的考勤、课前预习、课堂互动和课后作 业实现实时评价与考核。利用这一技术, 教师能够轻松地对每组学生的任务解决方案、任务总结、 课后作业进行评分, 这些都可以充分利用碎片时间在手机上进行。这不仅促使学生注重平时的学习 和积累, 也极大地减轻了教师批改作业的工作量, 而且在课堂讨论中通过自动记录学生作答情况, 使教师能够将注意力全部放于课堂内容的教学而不必记录学生讨论得分。

\section{3 教学实践效果}

为了检验基于雨课堂的任务式教学新模式的教学效果, 我们对应化 16-1、2 合班采用了两段式 教学模式进行对比研究, 即课程前 16 学时采用雨课堂任务导向式教学模式, 后 16 学时采用传统讲 授的教学模式。设计并实施了结课时的学生问卷调查, 主要目的是了解新教学模式的实际运用效果, 包括学生对雨课堂和任务导向教学在各个教学环节的内容安排、教学方法和形式的主观感受、满意 程度、学生收获和建议等多方面的情况。问卷设计为单选和多选, 为了反映学生的不同见解, 还设 计了开放式问答。除此以外, 在采用新教学模式的课程前半段还和平行对照班级进行了定性对比。

\section{1 数据收集及说明}

问卷的编制使用雨课堂的投票形式, 问卷发放和回收均采用雨课堂的试卷发布功能完成。调查 问卷选用匿名投票形式, 以便反映学生真实意愿。投票人数及选项分布由雨课堂系统自动统计。应 化 16-1、2 合班总人数 61 人, 回收调查问卷 55 份, 有效问卷 55 份。

\section{2 问卷调查结果与分析}

表 2 汇总了应化 16-1、2 班的问卷调查结果, 总体来说, 学生对雨课堂的授课形式、任务导向 的教学策略、作业评改、记分考核方式等方面的满意度还是很高的。下面是对各项反馈的具体分析。

首先, 学生认为本课程具有一定难度, 认为简单的比例仅占 $1.8 \%$, 因此教师改变教学策略、促 进学生学习具有必要性。在任务导向式及其与雨课堂融合方面, 超过 $87 \%$ 的学生认为课前推送任务 对预习帮助很大或较大, 超过 $81 \%$ 的学生认为融合雨课堂对完成任务非常有帮助或帮助较大, 无一 学生选阻碍任务完成。这表明任务导向式教学策略与雨课堂教学手段能够有机结合, 共同促进教学 效果的提高。

在课堂教学方面, 大多数学生反映传统课堂的问题主要是枯燥乏味和教师 “一言堂”, 因此促进 教学双方互动、提高课堂趣味性显得尤为重要。雨课堂与任务导向式教学融合后能够显著克服这些 缺点, 因此超过 $90 \%$ 的学生认为以雨课堂形式穿插课堂练习效果好, 采用雨课堂后, 近 $90 \%$ 的学生 认为课堂气氛变活跃、超过 $90 \%$ 学生认为学生参与程度和师生互动程度都提高了。

在记分考评方面, 超过 63\% 的学生反映利用雨课堂手段布置与完成作业效果较好, 但仍有近 $40 \%$ 学生觉得意义不大或更喜欢传统作业; 作业批改方式的问卷结果也显示大致相同的比例。而利用雨 课堂记录考勤、作业、测验等平时成绩时, $60 \%$ 的学生表示喜欢, $40 \%$ 的学生觉得一般或不喜欢。结 果表明, 雨课堂教学手段在日常教学管理方面也表现良好, 但还可以继续改进。 
表 2 问卷调查统计结果

\begin{tabular}{|c|c|c|c|c|c|}
\hline 设置的问题 & \multicolumn{5}{|c|}{ 选项及学生回答某选项的百分比 } \\
\hline \multirow[t]{2}{*}{ 1. 你认为本课程的内容 } & A 太难 & $\mathrm{B}$ 较难 & $\mathrm{C}$ 难度适中 & $\mathrm{D}$ 简单 & \\
\hline & $5.5 \%$ & $41.8 \%$ & $50.9 \%$ & $1.8 \%$ & \\
\hline 2.你认为课前布置任务对 & A 帮助很大 & $B$ 帮助较大 & $\mathrm{C}$ 帮助一般 & D 影响预习 & \\
\hline 预习 & $29.1 \%$ & $58.2 \%$ & $10.9 \%$ & $1.8 \%$ & \\
\hline 3.你怎么看通过雨课堂完 & A 非常有帮助 & $B$ 帮助较好 & C 无显效 & D 阻碍任务 & \\
\hline 成学习任务 & $27.3 \%$ & $54.5 \%$ & $18.2 \%$ & $0 \%$ & \\
\hline 4. 你认为传统课堂教学的 & A 教师一言堂 & $\mathrm{B}$ 枯燥乏味 & $\mathrm{C}$ 没机会展示自己 & $\mathrm{D}$ 迟到旷课较多 & $\mathrm{E}$ 睡觉玩手机较多 \\
\hline 问题是(多选) & $43.6 \%$ & $65.5 \%$ & $14.5 \%$ & $29.1 \%$ & $32.7 \%$ \\
\hline 5.你认为课堂穿插雨课堂 & A 很好 & B 较好 & $\mathrm{C}$ 一般 & D 不好 & \\
\hline 练习 & $52.7 \%$ & $40.0 \%$ & $5.5 \%$ & $1.8 \%$ & \\
\hline 6. 你认为通过雨课堂布置 & A 效率高 & B 比手写效果好 & $\mathrm{C}$ 与传统作业没差别 & D 不如传统作业 & \\
\hline 与完成作业 & $38.2 \%$ & $25.5 \%$ & $16.4 \%$ & $20.0 \%$ & \\
\hline 7.相对于教师批改作业方 & A 雨课堂方式 & B 教师全批改 & $\mathrm{C}$ 教师选批改 & D 无所谓 & \\
\hline 式, 你更喜欢 & $49.1 \%$ & $10.9 \%$ & $23.6 \%$ & $16.4 \%$ & \\
\hline 8. 采用雨课堂考核平时成 & $\mathrm{A}$ 很喜欢 & $\mathrm{B}$ 较喜欢 & $\mathrm{C}$ 一般 & $\mathrm{D}$ 不喜欢 & \\
\hline 绩 & $16.4 \%$ & $43.6 \%$ & $29.1 \%$ & $10.9 \%$ & \\
\hline 9.你认为本课程采用雨课 & A 显著活跃 & B 较大活跃 & $\mathrm{C}$ 有所活跃 & $\mathrm{D}$ 没有影响 & E下降 \\
\hline 堂教学总体上课堂气氛 & $14.5 \%$ & $43.6 \%$ & $30.9 \%$ & $9.1 \%$ & $1.8 \%$ \\
\hline 10.你认为本课程采用雨课 & A 显著变好 & $\mathrm{B}$ 较大变好 & $\mathrm{C}$ 有所变好 & $\mathrm{D}$ 没有变化 & E 下降 \\
\hline 堂教学总体上学生参与 & $18.2 \%$ & $34.5 \%$ & $38.2 \%$ & $9.1 \%$ & $0 \%$ \\
\hline 11.你认为本课程采用雨课 & A 显著提高 & B 较大提高 & $\mathrm{C}$ 有所提高 & $\mathrm{D}$ 没有提高 & E 下降 \\
\hline 堂教学总体上师生互动 & $20.0 \%$ & $40.0 \%$ & $32.7 \%$ & $5.5 \%$ & $1.8 \%$ \\
\hline 12.本课程新教学模式哪方 & A 化学反应的理解 & $\mathrm{B}$ 专业词汇的掌握 & $\mathrm{C}$ 英文应用水平 & D 化学理论的理解 & E 其他 \\
\hline 面你觉得收获最大? & $21.8 \%$ & $27.3 \%$ & $23.6 \%$ & $14.5 \%$ & $12.7 \%$ \\
\hline 13. 本课程新教学模式对你 & $\mathrm{A}$ 能够独立解决学习问题 & $\mathrm{B}$ 掌握了解决问题的 & $\mathrm{C}$ 较大长进 & D 长进不大 & $\mathrm{E}$ 没有长进 \\
\hline 解决问题能力(多选) & $20 \%$ & 技巧 & $50.9 \%$ & $18.2 \%$ & $1.8 \%$ \\
\hline & & $36.4 \%$ & & & \\
\hline
\end{tabular}

总体满意度和能力提高方面, 大多数学生觉得自己通过基于雨课堂的任务导向式教学, 解决问 题能力得到较大提高, $36 \%$ 以上的学生掌握了解决问题的技巧; 而收获最大方面, 学生选择最多的 前两项是专业词汇的掌握和英文应用水平的提高, 符合本课程的教学目标。

\section{3 与平行对照班的定性对比}

相对于对照班, 采用雨课堂与任务导向相融合的教学模式班级的学生对化学专业英语这门课程 的学习热情和学习效果都有了显著提高。表现为大部分学生课堂上不再埋头沉默, 而是积极踊跃地 报告自己的任务成果; 小部分学有余力的学生甚至不满足于课本的学习, 课余开始阅读英文化学学 术期刊。对比期中测验平均成绩, 显示采用新教学模式的班级成绩较对照班级有较大提高。 


\section{4 结语}

本文的研究发现, 在专业英语教学中任务导向与雨课堂的融合能够相互配合引导学生进入一条 循序渐进、反馈与提高的合理学习路径。二者结合的教学新模式能够显著地提高课堂气氛、师生互 动度和学生参与度, 融合雨课堂能显著提高任务教学的效果, 也能一定程度上改善日常教学管理, 该教学模式对学生兴趣和学业成绩的提高具有促进作用。因此, 它对传统教学模式是一个改革与补 充, 合理调整这两种模式使之更加灵活多样地应用于教学实践, 更加满足化学专业人才培养的需求, 这对实现 “人才梦、中国梦” 具有现实意义。

\section{参 考 文 献}

[1] 和媛, 张桢, 谢钢. 大学化学, 2018, 33 (11), 92 .

[2] 吴金风, 张丽, 马恒昌, 杨云霞. 化学教育, 2016, 37 (4), 5 .

[3] 缪素琴, 单永奎, 杨平, 杨帆. 化学教育, 2015, 36 (8), 20

[4] 冯欣, 李园园. 西部素质教育, 2018, 5 (10), 153.

[5] 许天旱, 王党会, 姚婷珍, 肖美霞. 高教学刊, 2017, No. 10, 2.

[6] 徐盛夏. 教育现代化, 2016, No. 35, 191.

[7] 王致华. 中国成人教育, 2007, No. 12, 166 .

[8] 田含笑. 创新教育, 2014, No. 7, 176. 